1 Hacettepe Journal of Mathematics and Statistics

$\bigcap$ Volume 46 (5) (2017), 851-863

\title{
On fuzzy soft semi-pre-open sets and fuzzy soft semi-pre-continuous mappings
}

\author{
Sabir Hussain *
}

\begin{abstract}
The main aim of this paper is to initiate and explore the properties of fuzzy soft semi-pre-open(closed) sets. We introduce and investigate fuzzy soft semi-pre-interior and fuzzy soft semi-pre-closure in fuzzy soft topological spaces. Moreover, we define and study the characterizations of fuzzy soft semi-pre-continuous and fuzzy soft semi-pre-open(closed) mappings in fuzzy soft topological spaces.
\end{abstract}

Keywords: Fuzzy soft sets, Fuzzy soft topology, Fuzzy soft semi-pre-open(closed), Fuzzy soft semi-pre-interior(closure), Fuzzy soft semi-pre-continuous, Fuzzy soft semi-pre-open(closed) mappings.

Received : 04.10.2016 Accepted: 04.01.2017 Doi : 10.15672/HJMS.2017.428

\section{Introduction}

D. Molodtsov [31] presented the concept of soft set as a new mathematical tool to solve varieties of complicated problems having uncertainties in real life, economics, engineering and compute sciences, social and medical sciences etc. In [32], D. Molodtsov et. al discussed the applications of soft sets in different fields. P. K. Maji et. al [27-28] explored the fundamental concepts of soft set theory in detail and established the applications of soft sets in decision making problems.

F. Feng et.al [12] introduced the soft product in soft set theory. Moreover, they gave generalization of uni-int decision making scheme in [13]. The relation of soft sets in information systems, criteria of measuring the sound quality, normal parameter reduction and classification of natural texture have been studied in [35],[26],[39], [25] and [33]. M. Shabbir and M. Naz [36] defined and discussed the basic concepts of soft topological spaces. Later on, many researchers [1], [3-4],[9-11],[14],[17-20],[30],[41] explored different algebraic structures of soft topological spaces.

B. Chen [6-7] defined and discussed soft semi-open(closed) sets in soft topological spaces. S. Hussain [21] continued to study the algebraic structures of soft semi-open(closed) sets. L. A. Zadeh [40] initiated the concept of fuzzy soft sets and provided a natural base to

*Department of Mathematics, College of Science, Qassim University, P. O. Box 6644, Buraydah 51482, Saudi Arabia.

Email : sabiriub@yahoo.com; sh.hussain@qu.edu.sa 
handle and improve mathematically the fuzzy phenomenon found in different areas of knowledge. C. L. Chang [5] studied and discussed fuzzy topological spaces. B. Ahmad and A. Kharral [2] investigated fuzzy soft sets and established fuzzy soft functions on fuzzy soft classes. The work on fuzzy soft topology improved further in [15-16],[24],[29], [34],[37-38].

In 2015, S. Hussain [22], initiated and explored the fuzzy soft semi-open(closed) sets by combining fuzzy soft sets and soft semi-open sets. S. Hussain investigated the properties of fuzzy soft semi-interior(closure), fuzzy soft semi-exterior, fuzzy soft semi-boundary, fuzzy soft semi-continuous and fuzzy soft semi-open(closed) mapping. Recently in 2016, S. Hussain [23] introduced and examined the basic properties of fuzzy soft $\alpha$-open(closed) sets, fuzzy soft pre-open(closed) sets, fuzzy soft regular-open(closed) sets and fuzzy soft neighborhood at fuzzy soft point. Moreover. S. Hussain analyzed the relationship between these notions.

\section{Preliminaries}

First we recall some definitions and results which will use in the sequel.

Definition 2.1[40]. A fuzzy set $f$ on $X$ is a mapping $f: X \rightarrow I=[0,1]$. The value $f(x)$ represents the degree of membership of $x \in X$ in the fuzzy set $f$, for $x \in X$.

Definition 2.2[31]. Let $X$ be an initial universe and $E$ be a set of parameters. Let $P(X)$ denotes the power set of $X$ and $A$ be a non-empty subset of $E$. A pair $(F, A)$ is called a soft set over $X$, where $F$ is a mapping given by $F: A \rightarrow P(X)$. In other words, a soft set over $X$ is a parameterized family of subsets of the universe $X$. For $e \in A, F(e)$ may be considered as the set of $e$-approximate elements of the soft set $(F, A)$.

Definition. 2.3[27]. Let $I^{X}$ denotes the set of all fuzzy sets on $X$ and $A \subseteq X$. A pair $(f, A)$ is called a fuzzy soft set over $X$, where $f: X \rightarrow I^{X}$ is a function. That is, for each $a \in A, f(a)=f_{a}: X \rightarrow I$, is a fuzzy set on $X$.

Definition 2.4[29]. For two fuzzy soft sets $(f, A)$ and $(g, B)$ over a common universe $X$, we say that $(f, A)$ is a fuzzy soft subset of $(g, B)$ if

(1) $A \subseteq B$ and

(2) for all $a \in A, f_{a} \leq g_{a}$; implies $f_{a}$ is a fuzzy subset of $g_{a}$.

We denote it by $(f, A) \tilde{\leq}(g, B)$. $(f, A)$ is said to be a fuzzy soft super set of $(g, B)$, if $(g, B)$ is a fuzzy soft subset of $(f, A)$. We denote it by $(f, A) \tilde{\geq}(g, B)$.

Definition 2.5[29]. Two fuzzy soft sets $(f, A)$ and $(g, B)$ over a common universe $X$ are said to be fuzzy soft equal, if $(f, A)$ is a fuzzy soft subset of $(g, B)$ and $(g, B)$ is a fuzzy soft subset of $(f, A)$.

Definition 2.6[29]. The union of two fuzzy soft sets of $(f, A)$ and $(g, B)$ over the common universe $X$ is the fuzzy soft set $(h, C)$, where $C=A \cup B$ and for all $c \in C$,

$$
h_{c}=\left\{\begin{array}{cl}
f_{c}, & \text { if } c \in A-B \\
g_{c}, & \text { if } c \in B-A \\
f_{c} \vee g_{c}, & \text { if } c \in A \cap B
\end{array}\right.
$$

We write $(f, A) \tilde{V}(g, B)=(h, C)$.

Definition 2.7[29]. The intersection $(h, C)$ of two fuzzy soft sets $(f, A)$ and $(g, B)$ over a common universe $X$, denoted $(f, A) \tilde{\wedge}(g, B)$, is defined as $C=A \cap B$, and $h_{c}=f_{c} \wedge g_{c}$, for all $c \in C$.

Definition 2.8[29]. The relative complement of a fuzzy soft set $(f, A)$ is the fuzzy soft set $\left(f^{c}, A\right)$, which is denoted by $(f, A)^{c}$, where $f^{c}: A \rightarrow F(U)$ is a fuzzy set-valued function. That is, for each $a \in A, f^{c}(A)$ is a fuzzy set in $U$, whose membership function is $f_{a}^{c}(x)=1 \tilde{\sim} f_{a}(x)$, for all $x \in U$. Here $f_{a}^{c}$ is the membership function of $f^{c}(a)$. 
Definition 2.9[16]. The difference $(h, C)$ of two fuzzy soft sets $(f, A)$ and $(g, B)$ over $X$, denoted by $(f, A) \tilde{\Upsilon}(g, B)$, is defined as $(f, A) \tilde{\Upsilon}(g, B)=(f, A) \tilde{\wedge}(g, B)^{c}$.

For our convenience, we will use the notation $f_{A}$ for fuzzy soft set instead of $(f, A)$.

Definition 2.10[37]. Let $\tau$ be the collection of fuzzy soft sets over $X$, then $\tau$ is said to be a fuzzy soft topology on $X$ if

(1) $\tilde{0}_{A}, \tilde{1_{A}}$ belong to $\tau$.

(2) If $\left(f_{A}\right)_{i} \in \tau$, for all $i \in I$, then $\tilde{\bigvee}_{i \in I}\left(f_{A}\right)_{i} \in \tau$.

(3) $f_{a}, g_{b} \in \tau$ implies that $f_{a} \tilde{\bigwedge} g_{b} \tilde{\in} \tau$.

The triplet $(X, \tau, A)$ is called a fuzzy soft topological space over $X$. Every member of $\tau$ is called fuzzy soft open set. A fuzzy soft set is called fuzzy soft closed if and only if its complement is fuzzy soft open.

Definition 2.11[38]. Let $(X, \tau, A)$ be a fuzzy soft topological space over $X$ and $f_{A}$ be a fuzzy soft set over $X$. Then

(1) fuzzy soft interior of fuzzy soft set $f_{A}$ over $X$ is denoted by $\left(f_{A}\right)^{\circ}$ and is defined as the union of all fuzzy soft open sets contained in $f_{A}$. Thus $\left(f_{A}\right)^{\circ}$ is the largest fuzzy soft open set contained in $f_{A}$.

(2) fuzzy soft closure of $f_{A}$, denoted by $\overline{f_{A}}$ is the intersection of all fuzzy soft closed super sets of $f_{A}$. Clearly $\bar{f}_{A}$ is the smallest fuzzy soft closed set over $\mathrm{X}$ which contains $f_{A}$.

Definition 2.12 [22]. Let $(X, \tau, A)$ be a fuzzy soft topological space over $X$. A fuzzy soft set $f_{A}$ is called fuzzy soft semi-open, if there exists a fuzzy soft open set $g_{A}$ such that $g_{A} \leq f_{A} \leq \overline{g_{A}}$. The class of all fuzzy soft semi-open sets in $X$ is denoted by $\operatorname{FSSO}(\mathrm{X})$. Note that every fuzzy soft open set is fuzzy soft semi-open but the converse is not true in general.

Definition 2.13[22]. A fuzzy soft set $f_{A}$ in fuzzy soft topological space $(X, \tau, A)$ is fuzzy soft semi-closed if and only if its complement $\left(f_{A}\right)^{c}$ is fuzzy soft semi-open. The class of fuzzy soft semi-closed sets is denoted by $\operatorname{FSSC}(\mathrm{X})$.

Note that every fuzzy soft closed set is fuzzy soft semi-closed in fuzzy soft topological space $(X, \tau, A)$.

Proposition 2.14[22]. Let $f_{A}$ be a fuzzy soft set in fuzzy soft topological space $(X, \tau, A)$. Then $f_{A}$ is fuzzy soft semi-closed if and only if there exists a fuzzy soft closed set $h_{A}$ such that $\left(h_{A}\right)^{0} \tilde{\leq} f_{A} \tilde{\leq} h_{A}$.

Definition 2.15[22]. Let $f_{A}$ be a fuzzy soft set in fuzzy soft topological space $(X, \tau, A)$. The fuzzy soft semi-closure of $f_{A}$, denoted by $s c l^{f s}\left(f_{A}\right)$ and is defined as the intersection of all fuzzy soft semi-closed supersets of $f_{A}$.

It is clear from the definition that $s c l^{f s}\left(f_{A}\right)$ is the smallest fuzzy soft semi-closed set over $X$ which contains $f_{A}$.

Definition 2.16[22]. Let $f_{A}$ be a fuzzy soft set in fuzzy soft topological space $(X, \tau, A)$. The fuzzy soft semi-interior of $f_{A}$, denoted by $\operatorname{sint}^{f s}\left(f_{A}\right)$ and is defined as the union of all fuzzy soft semi-open subsets of $f_{A}$.

It is clear from the definition that $\operatorname{sint}^{f s}\left(f_{A}\right)$ is the largest fuzzy soft semi-open set over $X$ contained in $f_{A}$.

Definition 2.17[23]. Let $(X, \tau, A)$ be a fuzzy soft topological space over $X$. Then a fuzzy soft set $f_{A}$ over $X$ is said to be a fuzzy soft pre-open, if $f_{A} \tilde{\leq}\left(\overline{f_{A}}\right)^{\circ}$.

Definition 2.18[23]. Let $(X, \tau, A)$ be a fuzzy soft topological space over $X$. Then a fuzzy soft set $f_{A}$ over $X$ is said to be a fuzzy soft pre-closed, if $\overline{\left(f_{A}\right)^{\circ}} \tilde{\leq} f_{A}$. 


\section{Fuzzy soft semi-pre-open(closed) sets}

Definition 3.1. Let $(X, \tau, A)$ be a fuzzy soft topological space over $X$, where $X$ is a nonempty set and $\tau$ is a family of fuzzy soft sets. Then a fuzzy soft set $f_{A}$ is said to be a fuzzy soft semi-pre-open, if there exists a fuzzy soft pre-open set $g_{A}$ such that $g_{A} \tilde{\leq} f_{A} \tilde{\leq} \overline{\left(g_{A}\right)}$.

Definition 3.2. Let $(X, \tau, A)$ be a fuzzy soft topological space over $X$, where $X$ is a nonempty set and $\tau$ is a family of fuzzy soft sets. Then a fuzzy soft set $f_{A}$ is said to be a fuzzy soft semi-pre-closed, if there exists a fuzzy soft pre-closed set $g_{A}$ such that $\left(g_{A}\right)^{\circ} \tilde{\leq} f_{A} \tilde{\leq} g_{A}$.

Note that the fuzzy soft set $f_{A}$ is fuzzy soft semi-pre-open if and only if $f_{A}^{c}$ is fuzzy soft semi-pre-closed.

Remark 3.3. It is clear that any fuzzy soft semi-open as well as fuzzy soft pre-open set is a fuzzy soft semi-pre-open set.

The following example shows that the converse of above remark is not true in general. For this we consider the Example 3.3[22] as:

Example 3.4. Let $X=\left\{h_{1}, h_{2}, h_{3}\right\}, A=\left\{e_{1}, e_{2}\right\}$ and $\tau=\left\{\tilde{0}, \tilde{1},\left(f_{A}\right)_{1},\left(f_{A}\right)_{2},\left(f_{A}\right)_{3}\right.$, $\left.\left(f_{A}\right)_{4}\right\}$ where $\left(f_{A}\right)_{1},\left(f_{A}\right)_{2},\left(f_{A}\right)_{3},\left(f_{A}\right)_{4}$ are fuzzy soft sets over $X$, defined as follows $f_{1}\left(e_{1}\right)\left(h_{1}\right)=0.5, f_{1}\left(e_{1}\right)\left(h_{2}\right)=0.3, f_{1}\left(e_{1}\right)\left(h_{3}\right)=0.2$, $f_{1}\left(e_{2}\right)\left(h_{1}\right)=0.3, f_{1}\left(e_{2}\right)\left(h_{2}\right)=0.5, f_{1}\left(e_{2}\right)\left(h_{3}\right)=0.2$, $f_{2}\left(e_{1}\right)\left(h_{1}\right)=1, f_{2}\left(e_{1}\right)\left(h_{2}\right)=0, f_{2}\left(e_{1}\right)\left(h_{3}\right)=0.5$ $f_{2}\left(e_{2}\right)\left(h_{1}\right)=0.5, f_{2}\left(e_{2}\right)\left(h_{2}\right)=0.3, f_{2}\left(e_{2}\right)\left(h_{3}\right)=1$, $f_{3}\left(e_{1}\right)\left(h_{1}\right)=0.5, f_{3}\left(e_{1}\right)\left(h_{2}\right)=0, f_{3}\left(e_{1}\right)\left(h_{3}\right)=0.2$, $f_{3}\left(e_{2}\right)\left(h_{1}\right)=0.3, f_{3}\left(e_{2}\right)\left(h_{2}\right)=0.3, f_{3}\left(e_{2}\right)\left(h_{3}\right)=0.2$, $f_{4}\left(e_{1}\right)\left(h_{1}\right)=1, f_{4}\left(e_{1}\right)\left(h_{2}\right)=0.3, f_{4}\left(e_{1}\right)\left(h_{3}\right)=0.5$, $f_{4}\left(e_{2}\right)\left(h_{1}\right)=0.5, f_{4}\left(e_{2}\right)\left(h_{2}\right)=0.5, f_{4}\left(e_{2}\right)\left(h_{3}\right)=1$.

Then $\tau$ is a fuzzy soft topology on $X$ and hence $(X, \tau, A)$ is a fuzzy soft topological space over $X$.

Note that the fuzzy soft closed sets are $\left\{\left\{h_{0.5}, h_{0.7}, h_{0.8}\right\},\left\{h_{0.7}, h_{0.5}, h_{0.8}\right\}\right\},\left\{\left\{h_{0}, h_{1}, h_{0.5}\right\},\left\{h_{0.5}, h_{0.7}, h_{0}\right\}\right\}$, $\left\{\left\{h_{0.5}, h_{1}, h_{0.8}\right\},\left\{h_{0.7}, h_{0.7}, h_{0.8}\right\}\right\},\left\{\left\{h_{0}, h_{0.7}, h_{0.5}\right\},\left\{h_{0.5}, h_{0.5}, h_{0}\right\}\right\}, \tilde{1}$ and $\tilde{0}$.

Let us take fuzzy soft set $f_{A}$ over $X$ defined by $f\left(e_{1}\right)\left(h_{1}\right)=0.3, f\left(e_{1}\right)\left(h_{2}\right)=0.2, f\left(e_{1}\right)\left(h_{3}\right)=0.1$ $f\left(e_{2}\right)\left(h_{1}\right)=0.2, f\left(e_{2}\right)\left(h_{2}\right)=0.4, f\left(e_{2}\right)\left(h_{3}\right)=0.1$.

That is, $f_{A}=\left\{\left\{h_{0.3}, h_{0.2}, h_{0.1}\right\},\left\{h_{0.2}, h_{0.4}, h_{0.1}\right\}\right\}$. Then there exists fuzzy soft set $g_{A}$ over $X$ defined by

$g\left(e_{1}\right)\left(h_{1}\right)=0.2, g\left(e_{1}\right)\left(h_{2}\right)=0.1, g\left(e_{1}\right)\left(h_{3}\right)=0.1$, $g\left(e_{2}\right)\left(h_{1}\right)=0.2, g\left(e_{2}\right)\left(h_{2}\right)=0.3, g\left(e_{2}\right)\left(h_{3}\right)=0.1$.

That is, $g_{A}=\left\{\left\{h_{0.2}, h_{0.1}, h_{0.1}\right\},\left\{h_{0.2}, h_{0.3}, h_{0.1}\right\}\right\}$.

Since $g_{A} \tilde{\leq}\left(\overline{g_{A}}\right)^{\circ} \cong\left\{\left\{h_{0.5}, h_{0.3}, h_{0.2}\right\},\left\{h_{0.3}, h_{0.5}, h_{0.2}\right\}\right\}$, then $g_{A}$ is fuzzy soft pre-open set over $X$. Moreover, calculations show that

$g_{A} \tilde{\leq} f_{A} \tilde{\leq} \overline{g_{A}} \tilde{=}\left\{\left\{h_{0.5}, h_{0.7}, h_{0.8}\right\},\left\{h_{0.7}, h_{0.5}, h_{0.8}\right\}\right\}$. This implies that $f_{A}$ is fuzzy soft semipre-open set. But $f_{A}$ is not fuzzy soft semi-open set, since there does not exist any fuzzy soft open set $h_{A}$ such that $h_{A} \tilde{\leq} f_{A} \tilde{\leq} \overline{h_{A}}$. Moreover, $f_{A}$ is not a fuzzy soft pre-open set.

Lemma 3.5. Let $(X, \tau, A)$ be fuzzy soft topological space over $X$ and $f_{A}$ be a fuzzy soft set over $X$. Then $f_{A} \tilde{\leq} \overline{\left(f_{A}\right)^{\circ}}$ if and only if $\overline{f_{A}} \tilde{=} \overline{\left(f_{A}\right)^{\circ}}$.

Proof. $f_{A} \tilde{\leq} \overline{\left(f_{A}\right)^{\circ}}$ follows that $\overline{f_{A}} \tilde{\leq} \overline{\left(\overline{\left(f_{A}\right)^{\circ}}\right)} \tilde{=} \overline{\left(f_{A}\right)^{\circ}} \tilde{\leq} \overline{f_{A}}$. Therefore, $\overline{f_{A}} \tilde{=} \overline{\left(f_{A}\right)^{\circ}}$. The other inclusion follows similarly.

Using Proposition 3.4[22] and above Lemma 3.5, we have

Theorem 3.6. Let $(X, \tau, A)$ be a fuzzy soft topological space over $X$ and $f_{A}$ be a fuzzy 
soft set over $X$. Then the following statements are equivalent.

(1) $f_{A}$ is fuzzy soft semi-open.

(2) $f_{A} \tilde{\leq} \overline{\left(f_{A}\right)^{\circ}}$.

(3) $\overline{f_{A}} \stackrel{\sim}{=} \overline{\left(f_{A}\right)^{\circ}}$.

The proof of the following theorem follows directly by taking complements in above Theorem 3.6.

Theorem 3.7. Let $(X, \tau, A)$ be a fuzzy soft topological space over $X$ and $f_{A}$ be a fuzzy soft set over $X$.Then the following statements are equivalent.

(1) $f_{A}$ is fuzzy soft semi-closed.

(2) $\left(\overline{f_{A}}\right)^{\circ} \tilde{\leq} f_{A}$.

(3) $\left(\overline{f_{A}}\right)^{\circ} \stackrel{\sim}{=}\left(f_{A}\right)^{\circ}$.

Theorem 3.8. Let $(X, \tau, A)$ be a fuzzy soft topological space over $X$ and $f_{A}$ be a fuzzy soft set over $X$. Then the following hold.

(1) If $f_{A}$ is fuzzy soft semi-pre-open, then $f_{A} \tilde{\leq} \overline{\left(\overline{f_{A}}\right)^{\circ}}$.

(2) If $f_{A}$ is fuzzy soft semi-pre-closed, then $\left(\overline{\left(f_{A}\right)^{\circ}}\right)^{\circ} \tilde{\leq} f_{A}$.

Proof. (1) $f_{A}$ is fuzzy soft semi-pre-open implies that there exists a fuzzy soft pre-open set $g_{A}$ such that $g_{A} \tilde{\leq} f_{A} \tilde{\leq} \overline{\left(g_{A}\right)}$. This follows that $\overline{f_{A}} \tilde{=} \overline{g_{A}}$. Also $g_{A}$ is fuzzy soft pre-open set implies that $f_{A} \tilde{\leq} \overline{g_{A}} \tilde{\leq} \overline{\left(\overline{g_{A}}\right)^{\circ}} \tilde{\leq} \overline{\left(\overline{f_{A}}\right)^{\circ}}$. Therefore, $f_{A} \tilde{\leq} \overline{\left(\overline{f_{A}}\right)^{\circ}}$.

(2) This can be proved in the same way as (1).

The following theorem directly follows from Theorems 3.6, 3.7 and 3.8.

Theorem 3.9. Let $(X, \tau, A)$ be a fuzzy soft topological space over $X$ and $f_{A}$ be a fuzzy soft set over $X$. If $f_{A}$ is fuzzy soft semi-pre-open(closed) and fuzzy soft open(closed), then $f_{A}$ is fuzzy soft semi-open(closed).

The following lemma is easy to proof.

Lemma 3.10. Let $(X, \tau, A)$ be a fuzzy soft topological space over $X$ and $f_{A_{i}}$ be a family of fuzzy soft set over $X$. Then $\bigvee \overline{F_{A_{i}}} \tilde{\leq} \overline{\bigvee F_{A_{i}}}$. Moreover, for finite case, $\bigvee \overline{F_{A_{i}}} \tilde{=} \overline{\bigvee F_{A_{i}}}$ and $\bigvee\left(F_{A_{i}}\right)^{\circ} \stackrel{\sim}{=}\left(\bigvee F_{A_{i}}\right)^{\circ}$.

Theorem 3.11. Let $(X, \tau, A)$ be a fuzzy soft topological space over $X$. Then

(1) Arbitrary union of fuzzy soft semi-pre-open sets is a fuzzy soft semi-pre-open set.

(2) Arbitrary intersection of fuzzy soft semi-pre-closed sets is a fuzzy soft semi-pre-closed set.

Proof. Suppose that $f_{A_{i}}$ be a family of fuzzy soft semi-pre-open set in fuzzy soft topological space over $X$. Then for each $f_{A_{i}}$, there exists a fuzzy soft pre-open set $g_{A_{i}}$ such that $g_{A_{i}} \tilde{\leq} f_{A_{i}} \tilde{\leq} \overline{g_{A_{i}}}$. Using Lemma 3.10 and Theorem 3.10[23], we have $\bigvee g_{A_{i}} \tilde{\sim} \bigvee f_{A_{i}} \tilde{\Sigma} \bigvee \overline{g_{A_{i}}} \tilde{\leq} \overline{\left(\bigvee g_{A_{i}}\right)}$, where $\bigvee g_{A_{i}}$ is fuzzy soft pre-open set.

(2) This follows directly by taking complements in (1).

Theorem 3.12. Let $f_{A}$ be a fuzzy soft set in fuzzy soft topological space $(X, \tau, A)$ over

$X$. Then

(1) $\operatorname{scl}^{f s}\left(f_{A}\right) \tilde{=} f_{A} \tilde{\bigvee}\left(\overline{f_{A}}\right)^{\circ}$.

(2) $\operatorname{sint}^{f s}\left(f_{A}\right) \tilde{=} f_{A} \tilde{\bigwedge} \overline{\left(\left(f_{A}\right)^{\circ}\right)}$.

(3) $\operatorname{sint}^{f s}\left(s c l^{f s}\left(f_{A}\right)\right) \tilde{=} s c l^{f s}\left(f_{A}\right) \tilde{\Lambda} \overline{\left(\left(\overline{f_{A}}\right)^{\circ}\right)}$.

(4) $s c l^{f s}\left(\operatorname{sint} t^{f s}\left(f_{A}\right)\right) \tilde{=} \operatorname{sint} t^{f s}\left(f_{A}\right) \tilde{\bigvee}\left(\overline{\left(\left(f_{A}\right)^{\circ}\right)}\right)^{\circ}$.

Proof. (1)and (2) directly follows from the definitions of fuzzy soft interior(closure) and fuzzy soft semi-interior(closure).

(3) Using (2), we have, $\operatorname{sint}^{f s}\left(s c l^{f s}\left(f_{A}\right)\right) \tilde{=} s c l^{f s}\left(f_{A}\right) \tilde{\Lambda} \overline{\left(\left(s c l^{f s}\left(f_{A}\right)\right)^{\circ}\right)}$

$\tilde{=} s c l^{f s}\left(f_{A}\right) \tilde{\Lambda}\left(\left(f_{A} \tilde{\bigvee}\left(\overline{f_{A}}\right)^{\circ}\right)^{\circ}\right) \tilde{\geq} s c l^{f s}\left(f_{A}\right) \tilde{\Lambda} \overline{\left(\left(f_{A}\right)^{\circ} \tilde{\bigvee}\left(\overline{f_{A}}\right)^{\circ}\right)} \tilde{=} s c l^{f s}\left(f_{A}\right) \tilde{\Lambda} \overline{\left(\left(\overline{\left(f_{A}\right)}\right)^{\circ}\right)}$. 
Also, $\operatorname{sint}^{f s}\left(s c l^{f s}\left(f_{A}\right)\right) \tilde{=} s c l^{f s}\left(f_{A}\right) \tilde{\Lambda} \overline{\left(\left(s c l^{f s}\left(f_{A}\right)\right)^{\circ}\right)} \tilde{\leq} s c l^{f s}\left(f_{A}\right) \tilde{\Lambda} \overline{\left(\left(\overline{f_{A}}\right)^{\circ}\right)}$. This follows (3).

(4) Using (1), we have, scl ${ }^{f s}\left(\operatorname{sint} t^{f s}\left(f_{A}\right)\right) \tilde{=} \operatorname{sint} t^{f s}\left(f_{A}\right) \tilde{\bigvee}\left(\overline{\left(\operatorname{sint} f^{f s}\left(f_{A}\right)\right)}\right)^{\circ} \tilde{=}$

$\operatorname{sint}^{f_{s}}\left(f_{A}\right) \tilde{\bigvee} \overline{\left(\left(f_{A} \tilde{\Lambda} \overline{\left(\left(f_{A}\right)^{\circ}\right)}\right)^{\circ}\right)} \tilde{\leq} \operatorname{sint}^{f_{s}}\left(f_{A}\right) \tilde{\bigvee}\left(\overline{f_{A}} \tilde{\Lambda} \overline{\left(\left(f_{A}\right)^{\circ}\right)}\right)^{\circ} \tilde{=} \operatorname{sint}^{f_{s}}\left(f_{A}\right) \tilde{\bigvee}\left(\overline{\left(\left(f_{A}\right)^{\circ}\right)}\right)^{\circ}$.

Also, $s c l^{f s}\left(\operatorname{sint}^{f s}\left(f_{A}\right)\right) \tilde{=} \operatorname{sint}^{f s}\left(f_{A}\right) \tilde{\bigvee}\left(\overline{\left(\operatorname{sint}^{f s}\left(f_{A}\right)\right)}\right)^{\circ} \tilde{\geq} \operatorname{sint} t^{f s}\left(f_{A}\right) \tilde{\bigvee}\left(\overline{\left(\left(f_{A}\right)^{\circ}\right)}\right)^{\circ}$. This proves

(4). Hence the proof.

Theorem 3.13. Let $f_{A}$ and $g_{A}$ are fuzzy soft sets in fuzzy soft topological space $(X, \tau, A)$ over $X$. If $f_{A}$ is fuzzy soft semi-pre-open and $f_{A} \tilde{\leq} g_{A} \tilde{\leq} \overline{f_{A}}$, then $g_{A}$ is a fuzzy soft semipre-open set.

Proof. $f_{A}$ is fuzzy soft semi-pre-open implies that there exists a fuzzy soft pre-open set $k_{A}$ in $(X, \tau, A)$ such that $k_{A} \tilde{\leq} f_{A} \tilde{\leq} \overline{k_{A}}$. As $f_{A} \tilde{\leq} g_{A}, k_{A} \tilde{\leq} f_{A} \tilde{\leq} g_{A}$ follows that $k_{A} \tilde{\leq} g_{A}$. Moreover, $\overline{f_{A}} \tilde{\leq} \overline{\left(\overline{k_{A}}\right)} \tilde{=} \overline{k_{A}}$ implies that $g_{A} \tilde{\leq} \overline{k_{A}}$. Therefore, $k_{A} \tilde{\leq} g_{A} \tilde{\leq} \overline{k_{A}}$. Hence $g_{A}$ is fuzzy soft semi-pre-open.

Definition 3.14[23]. A fuzzy soft set $f_{A}$ is said to be a fuzzy soft point in fuzzy soft topological space $(X, \tau, A)$ denoted by $e\left(f_{A}\right)$, if for the element $e \tilde{\in} A, f(e) \tilde{\neq} \tilde{0}$ and $f\left(e^{c}\right) \tilde{=} \tilde{0}$, for all $e^{c} \in A \backslash\{e\}$.

Definition 3.15[23]. The fuzzy soft point $e\left(f_{A}\right)$ is said to be in the fuzzy soft set $g_{A}$, denoted by $e\left(f_{A}\right) \tilde{\epsilon} g_{A}$, if for the element $e \in A, f(e) \leq g(e)$. Clearly, every fuzzy soft set $g_{A}$ can be expressed as the union of all fuzzy soft points which belong to $g_{A}$.

Definition 3.16[23]. The fuzzy soft point $\left(e\left(f_{A}\right)\right)^{c}$ is called the complement of a fuzzy soft point $e\left(f_{A}\right)$, if for all $e^{c} \in A-\{e\}, f\left(e^{c}\right) \tilde{=} \tilde{0}$ and $f(e) \tilde{\neq} \tilde{0}$, for any element $e \tilde{\in} A$.

Example 3.17[23]. Let $X=\left\{h_{1}, h_{2}, h_{3}\right\}, A=\left\{e_{1}, e_{2}\right\}$ and consider the fuzzy soft set $\left(f_{A}\right)_{1}$ over $X$ is defined as follows:

$f_{1}\left(e_{1}\right)\left(h_{1}\right)=0.5, f_{1}\left(e_{1}\right)\left(h_{2}\right)=0.3, f_{1}\left(e_{1}\right)\left(h_{3}\right)=0.2$,

$f_{1}\left(e_{2}\right)\left(h_{1}\right)=0.3, f_{1}\left(e_{2}\right)\left(h_{2}\right)=0.5, f_{1}\left(e_{2}\right)\left(h_{3}\right)=0.2$,

That is $\left(f_{A}\right)_{1}=\left\{\left\{h_{0.5}, h_{0.3}, h_{0.2}\right\},\left\{h_{0.3}, h_{0.5}, h_{0.2}\right\}\right\}$. Then

$e\left(\left(f_{A}\right)_{1}\right) \tilde{=}\left\{e_{1}=\left\{h_{0.5}, h_{0.3}, h_{0.2}\right\}\right\}$ is a fuzzy soft point. Moreover the complement $\left(e\left(\left(f_{A}\right)_{1}\right)^{c}\right.$ of $e\left(\left(f_{A}\right)_{1}\right)$ is $\left(e\left(\left(f_{A}\right)_{1}\right)^{c} \cong\left\{e_{1}=\left\{h_{0.5}, h_{0.7}, h_{0.8}\right\}\right\}\right.$.

Remark 3.18[23]. Note that, if the soft point $e\left(f_{A}\right)$ is in the soft set $g_{A}$, then it is not necessary that the complement $\left(e\left(f_{A}\right)\right)^{c}$ is in the soft set $\left(g_{A}\right)^{c}$.

The following example verify the above remark.

Example 3.19[23]. Let $X=\left\{h_{1}, h_{2}, h_{3}\right\}, A=\left\{e_{1}, e_{2}\right\}$. Then it is to be noted that the fuzzy soft point $e\left(f_{A}\right) \tilde{=}\left\{e_{1}=\left\{h_{0.2}, h_{0.1}, h_{0.4}\right\}\right\}$ is contained in the fuzzy soft set $g_{A}=\left\{\left\{h_{0.5}, h_{0.3}, h_{0.6}\right\},\left\{h_{0.3}, h_{0.5}, h_{0.2}\right\}\right\}$. Now we can see that the complement of fuzzy

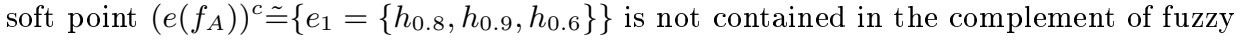
soft set $\left(g_{A}\right)^{c}=\left\{\left\{h_{0.5}, h_{0.7}, h_{0.4}\right\},\left\{h_{0.7}, h_{0.5}, h_{0.8}\right\}\right\}$.

Theorem 3.20. Let $f_{A}$ be a fuzzy soft set in fuzzy soft topological space $(X, \tau, A)$ over $X$. Then the following statements are equivalent:

(1) $f_{A}$ is fuzzy soft semi-pre-open.

(2) For every fuzzy soft point $e\left(g_{A}\right)$ in $f_{A}$, there exists a fuzzy soft semi-pre-open set $h_{A}$ such that $e\left(g_{A}\right) \tilde{\leq} h_{A} \tilde{\leq} f_{A}$.

Proof. $(1) \Rightarrow(2)$ Suppose that $f_{A}$ is fuzzy soft semi-pre-open. Then for every fuzzy soft point $e\left(g_{A}\right)$ in $f_{A}$, take $h_{A} \tilde{=} f_{A}$. We have $(2)$.

$(2) \Rightarrow(1)$ Consider $f_{A} \tilde{=} \tilde{V}_{e\left(g_{A}\right) \tilde{\epsilon} f_{A}}\left\{e\left(g_{A}\right)\right\} \tilde{\leq} \tilde{V}_{e\left(g_{A}\right) \tilde{\epsilon} f_{A}} h_{A} \tilde{\leq} f_{A}$. Hence the proof.

Theorem 3.21. Let $h_{A}$ and $k_{A}$ are fuzzy soft sets in fuzzy soft topological space $(X, \tau, A)$ over $X$. If $h_{A}$ is fuzzy soft semi-pre-closed with $\left(h_{A}\right)^{\circ} \tilde{\leq} k_{A} \tilde{\leq} h_{A}$, then $k_{A}$ is fuzzy soft semi-pre-closed.

Proof. This directly follows from Theorem 3.13.

Definition 3.22. Let $k_{A}$ be fuzzy soft set in fuzzy soft topological space $(X, \tau, A)$ over $X$ and $e\left(f_{A}\right)$ be a fuzzy soft point. If there exists a fuzzy soft semi-pre-open set $g_{A}$ with 
$e\left(f_{A}\right) \tilde{\epsilon} g_{A} \tilde{\leq} k_{A}$, then $k_{A}$ is called fuzzy soft semi-pre-neighborhood(nbd) of a fuzzy soft point $e\left(f_{A}\right)$.

The proof of the following theorem directly follows from the definitions of fuzzy soft point, fuzzy soft semi-pre-open and Theorem 3.11.

Theorem 3.23. Let $e\left(f_{A}\right)$ be a fuzzy soft point and $g_{A}$ be fuzzy soft set in fuzzy soft topological space $(X, \tau, A)$ over $X$. Then $g_{A}$ is fuzzy soft semi-pre-open if and only if $g_{A}$ is fuzzy soft semi-pre-nbd of $e\left(f_{A}\right)$, for every fuzzy soft point $e\left(f_{A}\right) \tilde{\in} g_{A}$.

Definition 3.24. Let $f_{A}$ be a fuzzy soft set in fuzzy soft topological space $(X, \tau, A)$ over $X$. Then

(1) fuzzy soft semi-pre-interior of fuzzy soft set $f_{A}$ denoted by $F^{s}$ pint $t^{s}$ and is defined as $F^{s} \operatorname{pint}^{s}\left(f_{A}\right) \tilde{=} \operatorname{Sup}\left\{g_{A}: g_{A}\right.$ is fuzzy soft semi-pre-open and $\left.g_{A} \tilde{\leq} f_{A}\right\}$.

(2) fuzzy soft semi-pre-closure of fuzzy soft set $f_{A}$ denoted by $F^{s} p c l^{s}$ and is defined as $F^{s} p c l^{s}\left(f_{A}\right) \tilde{=} \operatorname{Inf}\left\{g_{A}: g_{A}\right.$ is fuzzy soft semi-pre-closed and $\left.f_{A} \tilde{\leq} g_{A}\right\}$.

Theorem 3.25. Let $f_{A}$ be a fuzzy soft set in fuzzy soft topological space $(X, \tau, A)$ over $X$. Then

(1) $F^{s} p c l^{s}\left(f_{A}\right)^{c} \tilde{=}\left(F^{s} \operatorname{pint}^{s}\left(f_{A}\right)\right)^{c}$.

(2) $F^{s} \operatorname{pint}^{s}\left(f_{A}\right)^{c} \tilde{=}\left(F^{s} \text { pcl }^{s}\left(f_{A}\right)\right)^{c}$.

Proof. The proof follows form the Definition 3.24 and rule of complements.

Theorem 3.26. Let $e\left(f_{A}\right)$ be a fuzzy soft point and $g_{A}$ be fuzzy soft set in fuzzy soft topological space $(X, \tau, A)$ over $X$. Then $e\left(f_{A}\right) \tilde{\in} F^{s} p c l^{s}\left(g_{A}\right)$ if and only if $h_{A} \tilde{\leq} g_{A}$, for every fuzzy soft semi-pre-nbd $h_{A}$.

Proof. $(\Rightarrow)$ Contrarily suppose that there exists fuzzy soft semi-pre-nbd $h_{A}$ of $e\left(f_{A}\right)$ such

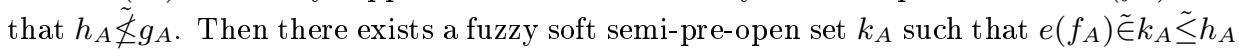
and $k_{A} \tilde{\leq} g_{A}$. Now $k_{A}^{c}$ is fuzzy soft semi-pre-closed set with $g_{A} \tilde{\leq} k_{A}$ implies that $F^{s} p c l^{s}\left(g_{A}\right) \tilde{\leq} k_{A}^{c}$. Also $e\left(f_{A}\right) \tilde{\in} k_{A}^{c}$ follows that $e\left(f_{A}\right) \tilde{\notin} F^{s} p c l^{s}\left(g_{A}\right)$. A contradiction.

$(\Leftarrow)$ Contrarily suppose that $e\left(f_{A}\right) \tilde{\notin} F^{s} p c l^{s}\left(g_{A}\right)$. Then there exists a fuzzy soft semi-preclosed set $l_{A}$ such that $e\left(f_{A}\right) \tilde{\notin} l_{A}$ and $g_{A} \tilde{\leq} l_{A}$. Therefore, $l_{A}^{c}$ is fuzzy soft semi-pre-open set such that $e\left(f_{A}\right) \tilde{\in} l_{A}^{c}$ with $g_{A} \tilde{\Varangle} l_{A}^{c}$. A contradiction. This completes the proof.

Theorem 3.27. Let $f_{A}$ be fuzzy soft set and $g_{A}$ be a fuzzy soft semi-pre-open set in fuzzy soft topological space $(X, \tau, A)$ over $X$. If $f_{A} \tilde{\not} g_{A}$, then $F^{s} p c l^{s}\left(f_{A}\right) \tilde{\not} g_{A}$.

Proof. Contrarily suppose that $F^{s} p c l^{s}\left(f_{A}\right) \tilde{\leq} g_{A}$. Then there exists a fuzzy soft point $e\left(h_{A}\right)$ in $(X, \tau, A)$ such that $F^{s} p c l^{s}\left(f_{\left.A_{(}\left(h_{A}\right)\right)}\right) \tilde{V}\left(g_{\left.A_{(}\left(h_{A}\right)\right)}\right) \tilde{\geq} \tilde{1}_{A}$. Take

$F^{s} p c l^{s}\left(f_{\left.A_{(} e\left(h_{A}\right)\right)}\right) \tilde{=} e\left(k_{A}\right)$. Then $g_{A}$ is a fuzzy soft semi-pre-nbd of $e\left(h_{A}\right)$ with respect to $e\left(k_{A}\right)$ such that $f_{A} \tilde{\not} g_{A}$. Therefore, $e\left(h_{A}\right) \tilde{\notin} F^{s} p c l^{s}\left(f_{A}\right)$. A contradiction. Hence the proof.

Theorem 3.28. Let $f_{A}$ be a fuzzy soft set in fuzzy soft topological space $(X, \tau, A)$ over $X$. Then $F^{s} p c l^{s}\left(f_{A}\right) \tilde{=} f_{A} \tilde{\bigvee}\left(\overline{\left(\left(f_{A}\right)^{\circ}\right)}\right)^{\circ}$.

Proof. Note that $\left\{\overline{\left(\left(f_{A} \tilde{\bigvee}\left(\overline{\left(\left(f_{A}\right)^{\circ}\right)}\right)^{\circ}\right)^{\circ}\right)}\right\}^{\circ} \tilde{\leq}\left\{\overline{\left(f_{A} \tilde{\bigvee}\left(\overline{\left(\left(f_{A}\right)^{\circ}\right)}\right)^{\circ}\right)}\right\}^{\circ} \tilde{\leq}\left\{\overline{\left(\left(f_{A}\right)^{\circ} \tilde{\bigvee} \overline{\left(\left(f_{A}\right)^{\circ}\right)}\right)}\right\}^{\circ}$ $\tilde{=}\left\{\overline{\left(\left(f_{A}\right)^{\circ}\right)}\right\}^{\circ} \tilde{=}\left(\overline{\left(\left(f_{A}\right)^{\circ}\right)}\right)^{\circ} \tilde{\leq} f_{A} \tilde{\leq}\left(\overline{\left(\left(f_{A}\right)^{\circ}\right)}\right)^{\circ}$. Therefore, $f_{A} \tilde{\bigvee}\left(\overline{\left(\left(f_{A}\right)^{\circ}\right)}\right)^{\circ}$ is fuzzy soft semipre-closed follows that $F^{s} p c l^{s}\left(f_{A}\right) \tilde{\leq} f_{A} \tilde{\bigvee}\left(\overline{\left(\left(f_{A}\right)^{\circ}\right)}\right)^{\circ}$.

Moreover, $F^{s} p c l^{s}\left(f_{A}\right)$ is fuzzy soft semi-pre-closed implies that $\left(\overline{\left(\left(f_{A}\right)^{\circ}\right)}\right)^{\circ} \tilde{\leq}\left(\overline{\left(\left(F^{s} p c l^{s}\left(f_{A}\right)\right)^{\circ}\right)}\right)^{\circ} \tilde{\leq} F^{s} p c l^{s}\left(f_{A}\right)$. Hence $f_{A} \tilde{\bigvee}\left(\overline{\left(\left(f_{A}\right)^{\circ}\right)}\right)^{\circ} \tilde{\leq} F^{s} p c l^{s}\left(f_{A}\right)$. This completes the proof.

Theorem 3.29. Let $f_{A}$ be a fuzzy soft set in fuzzy soft topological space $(X, \tau, A)$ over $X$. Then $F^{s} \operatorname{pint}^{s}\left(f_{A}\right) \tilde{=} f_{A} \tilde{\Lambda} \overline{\left(\left(\overline{f_{A}}\right)^{\circ}\right)}$.

Proof. Note that $f_{A} \tilde{\Lambda} \overline{\left(\left(\overline{f_{A}}\right)^{\circ}\right)} \tilde{\leq} \overline{\left(\left(\overline{f_{A}}\right)^{\circ}\right)} \tilde{=} \overline{\left(\left\{\overline{\left(f_{A}\right)} \tilde{\Lambda}\left(\overline{\left(f_{A}\right)}\right)^{\circ}\right\}^{\circ}\right)}$ 
$\left.\tilde{\leq} \overline{\left(\left\{\overline{\left(f_{A} \tilde{\Lambda}\left(\overline{\left(f_{A}\right)}\right)^{\circ}\right)}\right\}^{\circ}\right)} \tilde{\leq} \overline{\left(\left(\left\{f_{A} \tilde{\bigwedge} \overline{\left.\left(\overline{\left(f_{A}\right)}\right)^{\circ}\right)}\right\}\right)\right.}\right)^{\circ}$. Therefore, $f_{A} \tilde{\wedge} \overline{\left(\left\{\overline{\left(f_{A}\right)}\right\}^{\circ}\right)}$ is fuzzy soft semipre-open set. Hence $f_{A} \tilde{\bigwedge} \overline{\left(\left\{\overline{\left(f_{A}\right)}\right\}^{\circ}\right)} \tilde{\in} F^{s}$ pint $^{s}\left(f_{A}\right)$.

Also, since $F^{s} \operatorname{pint}^{s}\left(f_{A}\right)$ is fuzzy soft semi-pre-open, then

$F^{s}$ pint $^{s}\left(f_{A}\right) \tilde{\leq} \overline{\left(\left\{\overline{\left(F^{s} \text { pint }^{s}\left(f_{A}\right)\right)}\right\}^{\circ}\right)} \tilde{\leq} \overline{\left.\left(\overline{\left(f_{A}\right)}\right)^{\circ}\right)}$. Thus, $F^{s}$ pint $^{s}\left(f_{A}\right) \tilde{\leq} f_{A} \tilde{\bigwedge} \overline{\left(\left\{\left(\overline{\left(f_{A}\right)}\right)^{\circ}\right\}^{\circ}\right)}$. Hence the proof.

Theorem 3.30. Let $f_{A}$ be a fuzzy soft set in fuzzy soft topological space $(X, \tau, A)$ over

$X$. Then $F^{s} \operatorname{pint}^{s}\left(F^{s} p c l^{s}\left(f_{A}\right)\right) \simeq F^{s} p c l^{s}\left(F^{s} \operatorname{pint}^{s}\left(f_{A}\right)\right)$.

Proof. Using Theorems 3.28 and 3.29 , we have

$F^{s} \operatorname{pint}^{s}\left(F^{s} p c l^{s}\left(f_{A}\right)\right) \tilde{=} F^{s} p c l^{s}\left(f_{A}\right) \tilde{\Lambda} \overline{\left.\left(\overline{\left(F^{s} p c l^{s}\left(f_{A}\right)\right)}\right)^{\circ}\right)} \tilde{=}\left(f_{A} \tilde{\bigvee}\left(\overline{\left(\left(f_{A}\right)^{\circ}\right)}\right)^{\circ}\right) \tilde{\bigwedge} \overline{\left.\left(\overline{f_{A}}\right\}^{\circ}\right)}$

$\tilde{=}\left(f_{A} \tilde{\bigwedge} \overline{\left(\left\{\overline{f_{A}}\right\}^{\circ}\right)}\right) \tilde{\bigvee}\left\{\overline{\left(\left(f_{A}\right)^{\circ}\right)}\right\}^{\circ} \tilde{=} F^{s}$ pint $^{s}\left(f_{A}\right) \tilde{\bigvee}\left\{\overline{\left(\left\{F^{s} p_{i n t}\left(f_{A}\right)\right\}^{\circ}\right)}\right\}^{\circ} \tilde{=} F^{s} p c l^{s}\left(F^{s} p_{i n t}\left(f_{A}\right)\right)$.

This completes the proof.

Corollary 3.31. Let $f_{A}$ be a fuzzy soft set in fuzzy soft topological space $(X, \tau, A)$ over

$X$. Then

(1) $f_{A} \tilde{\bigvee} F^{s} p i n t^{s}\left(F^{s} p c l^{s}\left(f_{A}\right)\right) \tilde{=} F^{s} p c l^{s}\left(f_{A}\right)$.

(2) $f_{A} \tilde{\wedge} F^{s} \operatorname{pint}^{s}\left(F^{s} p c l^{s}\left(f_{A}\right)\right) \tilde{=} F^{s} \operatorname{pint}^{s}\left(f_{A}\right)$.

Theorem 3.32. Let $f_{A}$ be a fuzzy soft set in fuzzy soft topological space $(X, \tau, A)$ over $X$. Then, $s c l^{f s}\left(\operatorname{sint} t^{f s}\left(f_{A}\right)\right) \tilde{\leq} F^{s} p i n t^{s}\left(F^{s} p c l^{s}\left(f_{A}\right)\right) \tilde{\leq} s i n t^{f s}\left(s c l^{f s}\left(f_{A}\right)\right)$.

Proof. Using Theorems 3.12(3)(4) and 3.30, we get

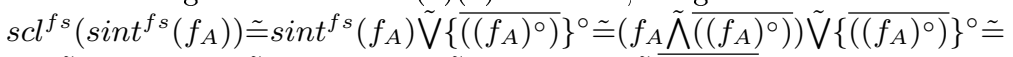

$\left(f_{A} \tilde{\bigvee}\left\{\overline{\left(\left(f_{A}\right)^{\circ}\right)}\right\}^{\circ}\right) \tilde{\Lambda} \overline{\left(\left(f_{A}\right)^{\circ}\right)} \tilde{\leq}\left(f_{A} \tilde{\bigvee}\left\{\overline{\left(\left(f_{A}\right)^{\circ}\right)}\right\}^{\circ}\right) \tilde{\Lambda} \overline{\left(\left(\overline{f_{A}}\right)^{\circ}\right)} \tilde{=} F^{s} \operatorname{pint}^{s}\left(F^{s} p c l^{s}\left(f_{A}\right)\right)$

$\tilde{\leq}\left(f_{A} \tilde{\bigvee}\left\{\overline{f_{A}}\right\}^{\circ}\right) \tilde{\Lambda} \overline{\left(\left(\overline{f_{A}}\right)^{\circ}\right)} \tilde{=} \operatorname{sint}^{f s}\left(\operatorname{scl}^{f s}\left(f_{A}\right)\right)$. Hence the proof.

The following theorem gives us the useful characterization of fuzzy soft semi-pre-open sets.

Theorem 3.33. Let $f_{A}$ be a fuzzy soft set in fuzzy soft topological space $(X, \tau, A)$ over

$X$. Then the following statements are equivalent.

(1) $f_{A}$ is fuzzy soft semi-pre-open.

(2) $f_{A} \tilde{\leq} F^{s} \operatorname{pint}^{s}\left(F^{s} p c l^{s}\left(f_{A}\right)\right)$.

(3) $f_{A} \tilde{\leq} \operatorname{sint} t^{f s}\left(s c l^{f s}\left(f_{A}\right)\right)$.

Proof. $(1) \Rightarrow(2)$ Suppose that $f_{A}$ be a fuzzy soft semi-pre-open set. Then

$f_{A} \stackrel{\sim}{=} F^{s}$ pint $^{s}\left(f_{A}\right) \tilde{\leq} F^{s}$ pint $^{s}\left(F^{s} p c l^{s}\left(f_{A}\right)\right)$.

$(2) \Rightarrow(3)$ This follows directly from Theorem 3.32.

$(3) \Rightarrow(1)$ Consider $f_{A} \tilde{\leq} s i n t^{f s}\left(s c l^{f s}\left(f_{A}\right)\right) \tilde{=} s c l^{f s}\left(f_{A}\right) \tilde{\Lambda} \overline{\left(\left(\overline{f_{A}}\right)^{\circ}\right)}$. This follows that $f_{A} \tilde{\leq} \overline{\left(\left(\overline{f_{A}}\right)^{\circ}\right)}$.

Hence $f_{A}$ is fuzzy soft semi-pre-open. This completes the proof.

The following theorem can be easily verified by using definitions.

Theorem 3.34 Let $f_{A}$ and $g_{A}$ are fuzzy soft set in fuzzy soft topological space $(X, \tau, A)$ over $X$. Then

(1) $F^{s} p c l^{s}\left(f_{A}\right) \tilde{\leq} \overline{f_{A}}$ and $\left(f_{A}\right)^{\circ} \tilde{\leq} F^{s} \operatorname{pint}^{s}\left(f_{A}\right)$.

(2) $F^{s}$ pcl $^{s}\left(f_{A}\right)\left(F^{s}\right.$ pint $\left.^{s}\left(f_{A}\right)\right)$ is fuzzy soft semi-pre-closed(open).

(3) $f_{A}$ is fuzzy soft semi-pre-closed(open) if and only if $F^{s} p c l^{s}\left(f_{A}\right) \tilde{=} f_{A}\left(F^{s} p i n t^{s}\left(f_{A}\right) \tilde{=} f_{A}\right)$.

(4) $f_{A} \tilde{\leq} g_{A}$ implies that $F^{s} p c l^{s}\left(f_{A}\right) \tilde{\leq} F^{s} p c l^{s}\left(g_{A}\right)$ and $F^{s} p i n t^{s}\left(f_{A}\right) \tilde{\leq} F^{s} p i n t^{s}\left(g_{A}\right)$.

\section{Fuzzy soft semi-pre-continuous functions}

In this section, we define and explore the characterizations of fuzzy soft semipre-continuous and fuzzy soft semi-pre-open(closed) mappings in a fuzzy soft topological spaces. First we recall some definitions. 
Definition 4.1[2]. Let $F(X, A)$ and $F(Y, B)$ be families of fuzzy soft sets. $u: X \rightarrow Y$ and $p: A \rightarrow B$ are mappings. Then image and inverse image of a function $f_{p u}$ : $F(X, A) \rightarrow F(Y, B)$ is defined as :

(1) Let $f_{A}$ be a fuzzy soft set in $F(X, A)$. The image of $f_{A}$ under $f_{p u}$, written as $f_{p u}\left(f_{A}\right)$, is a fuzzy soft set in $F(Y, B)$ such that for $\beta \in p(A) \subseteq B$ and $y \in Y$,

$$
\left\{\begin{array}{cc}
f_{p u}\left(f_{A}\right)(\beta)(y)= & \\
\bigvee_{x \in u^{-1}(y)}\left(\bigvee_{\alpha \in p^{-1}(\beta) \cap A}\left(f_{A}(\alpha)\right)(y),\right. & u^{-1}(y) \neq \phi, p^{-1}(\beta) \cap A \neq \phi \\
0, & \text { otherwise }
\end{array}\right.
$$

for all $y \in B . f_{p u}\left(f_{A}\right)$ is known as a fuzzy soft image of a fuzzy soft set $f_{A}$.

(2) Let $g_{B}$ be a fuzzy soft set in $F(Y, B)$. Then the fuzzy soft inverse image of $g_{B}$ under $f_{p u}$, written as $f_{p u}^{-1}\left(g_{B}\right)$, is a fuzzy soft set in $F(X, A)$ such that

$$
f_{p u}^{-1}\left(g_{B}\right)(\alpha)(x)=\left\{\begin{array}{cl}
g_{B}(p(\alpha))(u(x)), & p(\alpha) \in B \\
0, & \text { otherwise }
\end{array},\right.
$$

for all $x \in A$. $f_{p u}^{-1}\left(g_{B}\right)$ is known as a fuzzy soft inverse image of a fuzzy soft set $g_{B}$.

The fuzzy soft function $f_{p u}$ is called fuzzy soft surjective, if $p$ and $u$ are surjective. The fuzzy soft function $f_{p u}$ is called fuzzy soft injective, if $p$ and $u$ are injective.

Definition 4.2[23]. Let $\left(X, \tau_{1}, A\right)$ and $\left(Y, \tau_{2}, B\right)$ be two fuzzy soft topological spaces.

(1) A fuzzy soft mapping $f_{p u}: F(X, A) \rightarrow F(Y, B)$ is said to be fuzzy soft semicontinuous, if for any fuzzy soft open set $g_{B}$ in $\left(Y, \tau_{2}, B\right), f_{p u}^{-1}\left(g_{B}\right)$ is fuzzy soft semi-open in $\left(X, \tau_{1}, A\right)$.

(2) A fuzzy soft mapping $f_{p u}: F(X, A) \rightarrow F(Y, B)$ is said to be a fuzzy soft semi-open, if for any fuzzy soft open set $f_{A}$ in $\left(X, \tau_{1}, A\right), f_{p u}\left(f_{A}\right)$ is fuzzy soft semi-open in $\left(Y, \tau_{2}, B\right)$.

Now we define:

Definition 4.3. Let $\left(X, \tau_{1}, A\right)$ and $\left(Y, \tau_{2}, B\right)$ be two fuzzy soft topological spaces and $f_{p u}: F(X, A) \rightarrow F(Y, B)$ is a fuzzy soft mapping. Then $f_{p u}: F(X, A) \rightarrow F(Y, B)$ is said to be fuzzy soft semi-pre-continuous, if for every fuzzy soft open set $g_{B}$ in $\left(Y, \tau_{2}, B\right)$, $f_{p u}^{-1}\left(g_{B}\right)$ is fuzzy soft semi-pre-open in $\left(X, \tau_{1}, A\right)$.

Remark 4.4. Every fuzzy soft semi-continuous function is fuzzy soft semi-pre-continuous, since every fuzzy soft semi-open set is fuzzy soft semi-pre-open in fuzzy soft topological space. But the converse is not true in general.

The following theorem directly follows from Definition 4.3.

Theorem 4.5. Let $\left(X, \tau_{1}, A\right)$ and $\left(Y, \tau_{2}, B\right)$ be two fuzzy soft topological spaces and $f_{p u}: F(X, A) \rightarrow F(Y, B)$ is a fuzzy soft mapping. Then the following statements are equivalent:

(1) $f_{p u}$ is fuzzy soft semi-pre-continuous.

(2) For every fuzzy soft closed set $g_{B}$ in $\left(Y, \tau_{2}, B\right), f_{p u}^{-1}\left(g_{B}\right)$ is fuzzy soft semi-pre-closed in $\left(X, \tau_{1}, A\right)$.

Theorem 4.6. Let $\left(X, \tau_{1}, A\right)$ and $\left(Y, \tau_{2}, B\right)$ be two fuzzy soft topological spaces and $f_{p u}: F(X, A) \rightarrow F(Y, B)$ is a fuzzy soft mapping. Then the following statements are equivalent:

(1) $f_{p u}$ is fuzzy soft semi-pre-continuous.

(2) For every fuzzy soft point $e\left(f_{A}\right)$ in $\left(X, \tau_{1}, A\right)$ and every fuzzy soft open set $g_{A}$ with $f_{p u}\left(e\left(f_{A}\right)\right) \tilde{\epsilon} g_{A}$, there is fuzzy soft semi-pre-open set $h_{A}$ with $e\left(f_{A}\right) \tilde{\in} h_{A}$ and $f_{p u}\left(h_{A}\right) \tilde{\leq} g_{A}$.

Proof. $(1) \Rightarrow(2)$ Suppose that $e\left(f_{A}\right)$ be a fuzzy soft point in $\left(X, \tau_{1}, A\right)$ and $g_{A}$ be fuzzy 
soft open in $\left(Y, \tau_{2}, B\right)$ such that $f_{p u}\left(e\left(f_{A}\right)\right) \tilde{\in} g_{A}$. Take $h_{A} \tilde{=} f_{p u}^{-1}\left(g_{A}\right)$. Then by $(1), h_{A}$ is fuzzy soft semi-pre-open in $\left(X, \tau_{1}, A\right)$ with $e\left(f_{A}\right) \tilde{\in} h_{A}$ and $f_{p u}\left(h_{A}\right) \tilde{\leq} g_{A}$.

$(2) \Rightarrow(1)$ Suppose that $g_{A}$ be fuzzy soft open set in $\left(Y, \tau_{2}, B\right)$ and $e\left(f_{A}\right) \tilde{\in} f_{p u}^{-1}\left(g_{A}\right)$. Then $f_{p u}\left(e\left(f_{A}\right)\right) \tilde{\in} g_{A}$. By $(2)$, there is a fuzzy soft semi-pre-open set $h_{A}$ in $\left(X, \tau_{1}, A\right)$ such that $e\left(f_{A}\right) \tilde{\in} h_{A}$ and $f_{p u}\left(h_{A}\right) \tilde{\leq} g_{A}$. This follows that $e\left(f_{A}\right) \tilde{\in} h_{A} \tilde{\leq} f_{p u}^{-1}\left(g_{A}\right)$. Therefore, by Theorem 3.20, $f_{p u}^{-1}\left(g_{A}\right)$ is fuzzy soft semi-pre-open. Hence $f_{p u}$ is fuzzy soft semi-precontinuous. This completes the proof.

Definition 4.7[21]. Let $f_{A}$ be fuzzy soft set in fuzzy soft topological space $(X, \tau, A)$ over $X$ and $e\left(f_{A}\right)$ be fuzzy soft point. If there exists a fuzzy soft open set $g_{A}$ with $e\left(f_{A}\right) \tilde{\in} g_{A} \tilde{\leq} f_{A}$, then $f_{A}$ is called fuzzy soft neighborhood(nbd) of a fuzzy soft point $e\left(f_{A}\right)$. Theorem 4.8. Let $\left(X, \tau_{1}, A\right)$ and $\left(Y, \tau_{2}, B\right)$ be two fuzzy soft topological spaces and $f_{p u}: F(X, A) \rightarrow F(Y, B)$ is a fuzzy soft mapping. Then the following statements are equivalent:

(1) $f_{p u}$ is fuzzy soft semi-pre-continuous.

(2) For every fuzzy soft point $e\left(f_{A}\right)$ in $\left(X, \tau_{1}, A\right)$ and every fuzzy soft $\operatorname{nbd} g_{A}$ of $f_{p u}\left(e\left(f_{A}\right)\right)$, $f_{p u}^{-1}\left(g_{A}\right)$ is fuzzy soft semi-pre-nbd of $e\left(f_{A}\right)$.

(3) For every fuzzy soft point $e\left(f_{A}\right)$ in $\left(X, \tau_{1}, A\right)$ and every fuzzy soft $\operatorname{nbd} g_{A}$ of $f_{p u}\left(e\left(f_{A}\right)\right)$, there is fuzzy soft semi-pre-nbd $k_{A}$ of $e\left(f_{A}\right)$ such that $f_{p u}\left(k_{A}\right) \tilde{\leq} g_{A}$.

(4) For every fuzzy soft point $e\left(f_{A}\right)$ in $\left(X, \tau_{1}, A\right)$ and every fuzzy soft open set $g_{A}$ with $f_{p u}\left(e\left(f_{A}\right)\right) \tilde{\in} g_{A}$, there is fuzzy soft semi-pre-open set $h_{A}$ with $e\left(f_{A}\right) \tilde{\in} h_{A}$ and $f_{p u}\left(h_{A}\right) \tilde{\leq} g_{A}$. Proof. $(1) \Rightarrow(2)$ Suppose that $e\left(f_{A}\right)$ be a fuzzy soft point in $\left(X, \tau_{1}, A\right)$ and $g_{A}$ be a fuzzy soft nbd of $f_{p u}\left(e\left(f_{A}\right)\right)$. This follows that there is a fuzzy soft open set $k_{A}$ such that $f_{p u}\left(e\left(f_{A}\right)\right) \tilde{\leq} k_{A} \tilde{\leq} g_{A}$. Now $f_{p u}^{-1}\left(k_{A}\right)$ is fuzzy soft semi-pre-open and $e\left(f_{A}\right) \tilde{\leq} f_{p u}^{-1}\left(k_{A}\right) \tilde{\leq} f_{p u}^{-1}\left(g_{A}\right)$. Therefore $f_{p u}^{-1}\left(h_{A}\right)$ is fuzzy soft semi-pre-nbd of $e\left(f_{A}\right)$ in $\left(X, \tau_{1}, A\right)$.

$(2) \Rightarrow(3)$ Suppose that $e\left(f_{A}\right)$ be a fuzzy soft point in $\left(X, \tau_{1}, A\right)$ and $g_{A}$ be a fuzzy soft nbd of $f_{p u}\left(e\left(f_{A}\right)\right)$. This implies that $k_{A} \tilde{=} f_{p u}^{-1}\left(g_{A}\right)$ is fuzzy soft semi-pre-nbd of $e\left(f_{A}\right)$ and

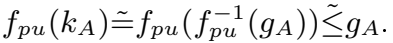

$(3) \Rightarrow(4)$ Let $e\left(f_{A}\right)$ be a fuzzy soft point in $\left(X, \tau_{1}, A\right)$ and $g_{A}$ be a fuzzy soft open set such that $f_{p u}\left(e\left(f_{A}\right)\right) \tilde{\epsilon} g_{A}$. Then $g_{A}$ is fuzzy soft nbd of $f_{p u}\left(e\left(f_{A}\right)\right)$. Therefore, there is fuzzy soft semi-pre-nbd $k_{A}$ of $e\left(f_{A}\right)$ in $\left(X, \tau_{1}, A\right)$ such that $e\left(f_{A}\right) \tilde{\in} k_{A}$ and $f_{p u}\left(k_{A}\right) \tilde{\leq} g_{A}$. Therefore, there is a fuzzy soft semi-pre-open set $h_{A}$ such that $e\left(f_{A}\right) \tilde{\in} h_{A} \tilde{\leq} k_{A}$. Hence $f_{p u}\left(h_{A}\right) \tilde{\leq} f_{p u}\left(k_{A}\right) \tilde{\leq} g_{A}$.

$(4) \Rightarrow(1)$ This follows from Theorem 4.6. Hence the proof.

Theorem 4.9. Let $\left(X, \tau_{1}, A\right)$ and $\left(Y, \tau_{2}, B\right)$ be two fuzzy soft topological spaces and $f_{p u}: F(X, A) \rightarrow F(Y, B)$ is a fuzzy soft mapping. Then the following statements are equivalent:

(1) $f_{p u}$ is fuzzy soft semi-pre-continuous.

(2) $f_{p u}^{-1}\left(\left(g_{B}\right)^{\circ}\right) \tilde{\Sigma} F^{s} p i n t^{s}\left(f_{p u}^{-1}\left(g_{B}\right)\right)$, for every fuzzy soft set $g_{B}$ in $\left(Y, \tau_{2}, B\right)$.

Proof. $(1) \Rightarrow(2)$ Suppose that $g_{B}$ be fuzzy soft set in $\left(Y, \tau_{2}, B\right)$. Then $\left(g_{B}\right)^{\circ}$ is fuzzy soft open set. Since $f_{p u}: F(X, A) \rightarrow F(Y, B)$ is fuzzy soft semi-pre-continuous, then $f_{p u}^{-1}\left(\left(g_{B}\right)^{\circ}\right)$ is fuzzy soft semi-pre-open in $\left(X, \tau_{1}, A\right)$. Therefore, $f_{p u}^{-1}\left(\left(g_{B}\right)^{\circ}\right) \tilde{\leq} f_{p u}^{-1}\left(g_{B}\right)$ implies that $f_{p u}^{-1}\left(\left(g_{B}\right)^{\circ}\right) \tilde{\leq} F^{s} p_{i n t}^{s}\left(f_{p u}^{-1}\left(g_{B}\right)\right)$.

$(2) \Rightarrow(1)$ This can be proved similarly. This completes the proof.

Theorem 4.10. Let $\left(X, \tau_{1}, A\right)$ and $\left(Y, \tau_{2}, B\right)$ be two fuzzy soft topological spaces and $f_{p u}: F(X, A) \rightarrow F(Y, B)$ be a fuzzy soft mapping. Then the following statements are equivalent:

(1) $f_{p u}$ is fuzzy soft semi-pre-continuous.

(2) $f_{p u}\left(F^{s} p c l^{s}\left(f_{A}\right)\right) \tilde{\leq} \overline{\left(f_{p u}\left(f_{A}\right)\right)}$, for every fuzzy soft set $f_{A}$ in $\left(X, \tau_{1}, A\right)$.

(3) $F^{s} p c l^{s}\left(f_{p u}^{-1}\left(g_{B}\right)\right) \tilde{\leq} f_{p u}^{-1}\left(\overline{g_{B}}\right)$, for every fuzzy soft set $g_{B}$ in $\left(Y, \tau_{2}, B\right)$.

Proof. $(1) \Rightarrow(2)$ Let $f_{A}$ be a fuzzy soft set in $\left(X, \tau_{1}, A\right)$. Then $\overline{\left(f_{p u}\left(f_{A}\right)\right)}$ is fuzzy soft 
closed. Since $f_{p u}$ is fuzzy semi-pre-continuous, then by Theorem 4.5, $f_{p u}^{-1}\left(\overline{\left(f_{p u}\left(f_{A}\right)\right)}\right)$ is fuzzy soft semi-pre-closed. Thus $f_{p u}^{-1}\left(\overline{\left(f_{p u}\left(f_{A}\right)\right)}\right) \stackrel{\sim}{=}$

$F^{s} p c l^{s}\left(f_{p u}^{-1}\left(\overline{\left(f_{p u}\left(f_{A}\right)\right)}\right)\right)$. Now $f_{A} \tilde{\leq} f_{p u}^{-1}\left(f_{p u}\left(f_{A}\right)\right)$ implies that $F^{s} p c l^{s}\left(f_{A}\right)$

$\tilde{\leq} F^{s} p c l^{s}\left(f_{p u}^{-1}\left(f_{p u}\left(f_{A}\right)\right)\right) \tilde{\leq} F^{s} p c l^{s}\left(f_{p u}^{-1}\left(f_{p u}\left(\overline{f_{A}}\right)\right)\right) \stackrel{\sim}{=} f_{p u}^{-1}\left(\overline{\left(f_{p u}\left(f_{A}\right)\right)}\right)$.

$(2) \Rightarrow(3)$ Let $g_{B}$ be fuzzy soft set in $\left(Y, \tau_{2}, B\right)$. Then by (2), we get

$f_{p u}\left(F^{s} p c l^{s}\left(f_{p u}^{-1}\left(g_{B}\right)\right)\right) \tilde{\leq} \overline{f_{p u}\left(f_{p u}^{-1}\left(g_{B}\right)\right)}$. Hence $F^{s} p c l^{s}\left(f_{p u}^{-1}\left(g_{B}\right)\right) \tilde{\leq} f_{p u}^{-1}\left(\overline{f_{p u}\left(f_{p u}^{-1}\left(g_{B}\right)\right)}\right)$

$\tilde{\leq} f_{p u}^{-1}\left(\overline{g_{B}}\right)$.

$(3) \Rightarrow(1)$ Let $g_{B}$ be fuzzy soft open set in $\left(Y, \tau_{2}, B\right)$. Then $g_{B}^{c}$ is fuzzy soft closed. By (3), we have $F^{s} p c l^{s}\left(f_{p u}^{-1}\left(g_{B}^{c}\right)\right) \tilde{\leq} f_{p u}^{-1}\left(\overline{g_{B}^{c}}\right) \tilde{=} f_{p u}^{-1}\left(g_{B}^{c}\right)$. Therefore, Theorem 3.25 follows that $f_{p u}^{-1}\left(g_{B}^{c}\right) \tilde{\geq} F^{s} p c l^{s}\left(f_{p u}^{-1}\left(g_{B}^{c}\right)\right) \simeq\left(F^{s} p^{\prime n n t} t^{s}\left(f_{p u}^{-1}\left(g_{B}\right)\right)\right)^{c}$. Hence $f_{p u}^{-1}\left(g_{B}\right)$ is fuzzy soft semi-preopen and therefore $f_{p u}$ is fuzzy soft semi-pre-continuous. Hence the proof.

Definition 4.11. Let $\left(X, \tau_{1}, A\right)$ and $\left(Y, \tau_{2}, B\right)$ be two fuzzy soft topological spaces. Then a fuzzy soft mapping $f_{p u}: F(X, A) \rightarrow F(Y, B)$ is said to be a fuzzy soft semi-open, if for any fuzzy soft open set $f_{A}$ in $\left(X, \tau_{1}, A\right), f_{p u}\left(f_{A}\right)$ is fuzzy soft semi-pre-open set in $\left(Y, \tau_{2}, B\right)$.

Remark 4.12. Every fuzzy soft semi-open function is fuzzy soft semi-pre-open, since every fuzzy soft semi-open set is fuzzy soft semi-pre-open. But the converse is not true in general.

Theorem 4.13. Let $\left(X, \tau_{1}, A\right)$ and $\left(Y, \tau_{2}, B\right)$ be two fuzzy soft topological spaces and $f_{A}$ be fuzzy soft set in $\left(X, \tau_{1}, A\right)$. Then a fuzzy soft mapping $f_{p u}: F(X, A) \rightarrow F(Y, B)$ is fuzzy soft semi-pre-open if and only $f_{p u}\left(\left(f_{A}\right)^{\circ}\right) \tilde{\Sigma} \operatorname{sint} t^{f s}\left(s c l^{f s}\left(f_{p u}\left(f_{A}\right)\right)\right)$.

Proof. $(\Rightarrow)$ Suppose $f_{p u}$ is fuzzy soft semi-pre-open and also for any fuzzy soft set $f_{A}$ in $\left(X, \tau_{1}, A\right)$, we get $f_{p u}\left(\left(f_{A}\right)^{\circ}\right) \tilde{\leq} f_{p u}\left(f_{A}\right)$. Our hypothesis follows that $f_{p u}\left(\left(f_{A}\right)^{\circ}\right)$ is fuzzy soft semi-pre-open in $\left(Y, \tau_{2}, B\right)$. Using Theorem 3.33, we have

$f_{p u}\left(\left(f_{A}\right)^{\circ}\right) \tilde{\Sigma} \operatorname{sint}^{f s}\left(s c l^{f s}\left(f_{p u}\left(f_{A}\right)\right)\right)$.

$(\Leftarrow)$ Suppose that $f_{p u}\left(\left(f_{A}\right)^{\circ}\right) \tilde{\leq} \operatorname{sint} t^{f s}\left(s c l^{f s}\left(f_{p u}\left(f_{A}\right)\right)\right)$, for fuzzy soft set $f_{A}$ in $\left(X, \tau_{1}, A\right)$. Then $g_{A}$ be any fuzzy soft open set in $\left(X, \tau_{1}, A\right)$. Now take $g_{A} \tilde{=}\left(f_{A}\right)^{\circ}$

$f_{p u}\left(g_{A}\right) \tilde{=} f_{p u}\left(\left(f_{A}\right)^{\circ}\right) \tilde{\dot{\leq}} \operatorname{sint} t^{f s}\left(s c l^{f s}\left(f_{A}\right)\right)$. This follows that $f_{p u}\left(g_{A}\right) \tilde{\check{\Sigma}}_{\operatorname{sint}} \operatorname{sis}^{f s}\left(s c l^{f s}\left(f_{p u}\left(f_{A}\right)\right)\right)$. Theorem 3.33 implies that $f_{p u}\left(g_{A}\right)$ is a fuzzy soft semi-pre-open in $\left(Y, \tau_{2}, B\right)$. Therefore, $f_{p u}$ is a fuzzy soft semi-open function. This completes the proof.

Theorem 4.14. Let $\left(X, \tau_{1}, A\right)$ and $\left(Y, \tau_{2}, B\right)$ be two fuzzy soft topological spaces. Then fuzzy soft mapping $f_{p u}: F(X, A) \rightarrow F(Y, B)$ is fuzzy soft semi-pre-open if and only $f_{p u}\left(f_{A}^{\circ}\right) \tilde{\leq} F^{s} p_{i n t}^{s}\left(f_{p u}\left(f_{A}\right)\right)$, for every fuzzy soft set $f_{A}$ in $\left(X, \tau_{1}, A\right)$.

Proof. $(\Rightarrow)$ Suppose that $f_{p u}$ is fuzzy soft semi-pre-open and $f_{A}$ be fuzzy soft set in $\left(X, \tau_{1}, A\right)$. Then $f_{p u}\left(f_{A}^{\circ}\right)$ is fuzzy soft semi-pre-open. Thus,

$f_{p u}\left(f_{A}^{\circ}\right) \tilde{=} F^{s} \operatorname{pint}^{s}\left(f_{p u}\left(f_{A}^{\circ}\right)\right) \tilde{\leq} F^{s} \operatorname{pint}^{s}\left(f_{p u}\left(f_{A}\right)\right)$. Hence $f_{p u}\left(f_{A}^{\circ}\right) \tilde{\leq} F^{s} p i n t^{s}\left(f_{p u}\left(f_{A}\right)\right)$.

$(\Leftarrow)$ Suppose that $f_{A}$ be a fuzzy soft open set in $\left(X, \tau_{1}, A\right)$. Then our hypothesis follows that $f_{p u}\left(f_{A}\right) \tilde{=} f_{p u}\left(f_{A}^{\circ}\right) \tilde{\leq} F^{s} p_{i n t}^{s}\left(f_{p u}\left(f_{A}\right)\right)$. Thus $f_{p u}\left(f_{A}\right)$ is fuzzy soft semi-pre-open in $\left(Y, \tau_{2}, B\right)$. Hence the proof.

Definition 4.15. Let $\left(X, \tau_{1}, A\right)$ and $\left(Y, \tau_{2}, B\right)$ be two fuzzy soft topological spaces. Then a fuzzy soft mapping $f_{p u}: F(X, A) \rightarrow F(Y, B)$ is said to be a fuzzy soft semi-pre-closed, if for any fuzzy soft closed set $f_{A}$ in $\left(X, \tau_{1}, A\right), f_{p u}\left(f_{A}\right)$ is fuzzy soft semi-pre-closed in $\left(Y, \tau_{2}, B\right)$.

Remark 4.16. Every fuzzy soft semi-closed function is fuzzy soft semi-pre-closed but the converse is not true in general, since every fuzzy soft semi-closed set is fuzzy soft semi-pre-closed [23].

Theorem 4.17. Let $\left(X, \tau_{1}, A\right)$ and $\left(Y, \tau_{2}, B\right)$ be two fuzzy soft topological spaces and $f_{A}$ be fuzzy soft set in $\left(X, \tau_{1}, A\right)$. Then a fuzzy soft mapping $f_{p u}: F(X, A) \rightarrow F(Y, B)$ is fuzzy soft semi-pre-closed if and only $F^{s} p c l^{s}\left(f_{p u}\left(f_{A}\right)\right) \tilde{\leq} f_{p u}\left(\overline{f_{A}}\right)$.

Proof. $(\Rightarrow)$ Let $f_{p u}$ be fuzzy soft semi-pre-closed and $f_{A}$ be fuzzy soft set in $\left(X, \tau_{1}, A\right)$. 
Then $f_{p u}\left(\overline{f_{A}}\right)$ is fuzzy soft semi-pre-closed in $\left(Y, \tau_{2}, B\right)$. Therefore, $f_{p u}\left(f_{A}\right) \tilde{\leq} f_{p u}\left(\overline{f_{A}}\right)$ follows that $F^{s} p c l^{s}\left(f_{p u}\left(f_{A}\right)\right) \tilde{\leq} f_{p u}\left(\overline{f_{A}}\right)$.

$(\Leftarrow)$ Suppose that $g_{A}$ be fuzzy soft closed in $\left(X, \tau_{1}, A\right)$. Then

$f_{p u}\left(g_{A}\right) \tilde{=} f_{p u}\left(\overline{g_{A}}\right) \tilde{\geq} F^{s} p c l^{s}\left(f_{p u}\left(g_{A}\right)\right)$. Also by definition of fuzzy soft semi-pre-closure, $f_{p u}\left(g_{A}\right) \tilde{\leq} F^{s} p c l^{s}\left(f_{p u}\left(g_{A}\right)\right)$. This implies that $f_{p u}\left(g_{A}\right) \tilde{=} F^{s} p c l^{s}\left(f_{p u}\left(g_{A}\right)\right)$. Therefore, $f_{p u}$ is a fuzzy soft semi-pre-closed mapping. This completes the proof.

Conclusion: The separation properties for fuzzy soft sets seem to be of special similarity to the problem of pattern discrimination. The fuzzification of soft set theory is very important topic in recent days. Every day new methods are developing in the literature using fuzzy soft sets from an imprecise mutiobserver data for problems of decision making. In this paper, We initiated and explored the properties of fuzzy soft semi-pre-open(closed) sets. We observed that any fuzzy soft semi-open as well as fuzzy soft pre-open set is a fuzzy soft semi-pre-open set. But the converse is not true. We introduced and investigated fuzzy soft semi-pre-interior and fuzzy soft semi-pre-closure in fuzzy soft topological spaces. Moreover, we defined and studied the characterization of fuzzy soft semi-pre-continuous and fuzzy soft semi-pre-open(closed) mappings in fuzzy soft topological spaces, which generalized fuzzy soft semi-continuous and fuzzy soft semi-open(closed) mappings. In future studies, we may develop more properties with applications of fuzzy soft sets in optimization problems as well as the problems of pattern discrimination.

\section{References}

[1] B. Ahmad, S. Hussain : On some structures of soft topology, Mathematical Sciences, 6(64) (2012), 7 Pages.

[2] B. Ahmad, A. Kharal: Mappings on fuzzy soft classes, Advances in Fuzzy Systems, (2009), Article ID 407890, 6 Pages.

[3] H. Aktas, N. Cagman: Soft sets and soft groups, Information Sciences, 177(13)(2007), $2726-2735$.

[4] M. I. Ali, F. Feng, X. Liu, W. K. Min, and M. Shabir: On some new operations in soft set theory, Computers and Mathematics with Applications, 57(9)(2009),1547-1553.

[5] C. L. Chang: Fuzzy topological spaces, Journal of Mathematical Analysis and Applications, 24(1)(1968), 182-190.

[6] B. Chen: Soft semi-open sets and related properties in soft topological spaces, Applied Mathematics Information Sciences, 7(1)(2013), 287-294.

[7] B. Chen: Soft local properties of soft semi-open sets, Discrete Dynamics in Nature and Society, Vol. 2013, Article ID 298032, 6 pages.

[8] S. Du, Q. Qin, Q. Wang, and B. Li: Fuzzy description of topological relations I: a unified fuzzy 9-intersection model, Proceedings of the 1st International Conference on Advances in Natural Computation (ICNC -05), vol. 3612 of Lecture Notes in Computer Science, pp. 1261-1273, Changsha, China, August 2005.

[9] M. J. Egenhofer and R. D. Franzosa: Point-set topological spatial relations, International Journal of Geographical Information Systems, 5(2)(1991), 161-174.

[10] M. J. Egenhofer and J. Herring: Categorizing binary topological relations between regions, lines and points in geographic databases, Tech. Rep., Department of Surveying Engineering, University of Maine, Orono, Me, USA, 1991.

[11] F. Feng, Y. B. Jun, X. Zhao: Soft semi rings, Computers and Mathematics with Applications, 56(10)(2008), 2621-2628.

[12] F. Feng, Y. M. Li: Soft subsets and soft product operations, Information Sciences, 232(2013), 44-57.

[13] F. Feng, Y. M. Li, N. Cagman: Generalized uni-int decision making scheme based on choice value soft sets, Europeon Journal of Operaion Research, 220(2012), 162-170. 
[14] F. Feng, M. Akram, B. Davvaz, V. Leoreanu-Fotea: Attribute analysis of information systems based on elementry soft implications, Knowledge Based Systems, 70(2014),281292.

[15] F. Feng, W. Pedrycz: On scalar products and decomposition theorems of fuzzy soft sets, Journal of Multi-valued Logic and Soft Computing, 25(2015), 45-80.

[16] C. Gundaz, S. Bayramov: Some results on fuzzy soft topological spaces, Mathematical Problems in Engineering, (2013), Article ID 935308, 10 Pages.

[17] S. Hussain, B. Ahmad : Some properties of soft topological spaces, Computers and Mathematics with Applications, 62(11) (2011) 4058-4067.

[18] S. Hussain, B. Ahmad : Soft separation axioms in soft topological spaces, Hacettepe Journal of Mathematics and Statistics, 44(3) (2015), 559-568.

[19] S. Hussain: A note on soft connectedness, Journal of Egyptian Mathematical Society, 23(1) (2015), 6-11.

[20] S. Hussain: On some soft functions, Mathematical Science Letters, 4(1) (2015), 55-61.

[21] S. Hussain: Properties of soft semi-open and soft semi-closed sets, Pensee Journal, $76(2)(2014), 133-143$.

[22] S. Hussain: On some generalized structures in fuzzy soft topological spaces, Information Science Letters, 4(3)(2015), 107-115.

[23] S. Hussain: On weak and strong forms of fuzzy soft open sets, Fuzzy Information and Engineering, (8)(2016), 451-463.

[24] A. Z. Khameneh, A. Kilicman and A. R. Salleh: Fuzzy soft boundary, Annals of Fuzzy Mathematics and Informatics, 8(5)(2014), 687-703.

[25] Z. Kong, L. Gao, L. Wong, S. Li: The normal parameter reduction of soft sets and its algorithm, J. Comp. Appl. Math., 21 (2008), 941-945.

[26] B. Kostek: Soft set approach to subjective assesment of sound quality, Fuzzy Systems Proceeding, 1998, IEEE World Congress on Computational Intelligence, 4-9 May, 669674. 1998. DOI: 10.1109/FUZZY.1998.687568.

[27] P. K. Maji, R. Biswas, A. R. Roy: Soft set theory, Computers and Mathematics with Applications, 45(4-5) (2003), 555-562.

[28] P . K. Maji, R. Biswas, A. R. Roy: An application of soft sets in a decision making problem, Computers and Mathematics with Applications, 44(8-9) (2002), 1077-1083.

[29] P. K. Maji, R. Biswas, A. R. Roy: Fuzzy soft sets, J. Fuzzy Math., 9(3)(2001), 589-602.

[30] W. K. Min: Soft sets over a common topological universe, Jouranl of Intelligent and Fuzzy Systems, 26:2099(2014).

[31] D. Molodtsov: Soft set theory first results, Computers and Mathematics with Applications, 37(4-5) (1999), $19-31$.

[32] D. Molodtsov, V.Y. Leonov, D.V. Kovkov: Soft sets technique and its application, Nechetkie Sistemy i Myagkie Vychisleniya, 9(1)(2006), 28-39.

[33] M. Mushrif, S. Sengupta, A. K. Ray: Texture classification using a novel, soft set theory based classification algorithm, Springer Berlin, Heidelberg , 254-264(2006).

[34] G. J. Nazaroff: Fuzzy topological polysystems, Journal of Mathematical Analysis and Applications, 41(1973), 734-742.

[35] D. Pie, D. Miao: From soft sets to information systems, X. Hu, Q. Liu, A. Skowron, Y. Y. Lin, R. R. Yager, B. Zhang(Eds.) Proceedings of Granular Computing Vol. 2, IEEE(2005), 2005, 617-621.

[36] M. Shabir, M. Naz: On soft topological spaces, Computers and Mathematics with Applications, 61(7)(2011), 1786-1799.

[37] B. Tanay and M. B. Kandemir: Topological structure of fuzzy soft sets, Computers and Mathematics with Applications, 61(10)(2011), 2952-295\%.

[38] B. P. Varol and H. Aygun: Fuzzy soft topology, Hacettepe Journal of Mathematics and Statistics, 41(3)(2012), 407-419.

[39] Z. Xio, L. Chen, B. Zhong, S. Ye: Recognition for information based on the theory of soft sets, J. Chen(Ed.), Procedding of ICSSSM-05, IEEE(2005), (2)(2005), 1104-1106.

[40] L. A. Zadeh: Fuzzy sets, Information and Control, 8(1996), 338-353.

[41] I. Zorlutana, N. Akdag, W. K. Min: Remarks on soft topological spaces, Annals of fuzzy Mathematics and Informatics, 3(2)(2012),171-185. 
